\title{
Cancer incidence among the south Asian and non-south Asian population under 30 years of age in Yorkshire, UK
}

M van Laar, P A McKinney, R C Parslow, A Glaser, S E Kinsey, I J Lewis, S V Picton, M Richards, G Shenton, D Stark, P Norman and R G Feltbower

Correction to: British Journal of Cancer (2010) 103, 1448-1452. doi:10.1038/sj.bjc.6605903

After publication of this article in 2010, it was noted that there was an error in the data format used for the analyses of incidence rates throughout the paper, which led to overestimation of the population denominators. The interpretation, $P$-values and main conclusions throughout the manuscript remain the same; however, it is the magnitude of the incidence rates that is incorrect. The correct data are given below.

Incidence rates

The incidence rate (per 1000000 person-years) for all cancers in 0-29 year olds between 1990 and 2005 is 151 (95\% CI 146-156).

Table 1. Number of cases and age-sex standardised incidence rates for south Asians and non-south Asians by diagnostic group, age group and gender (1990-2005)

Number of cases

Incidence rates

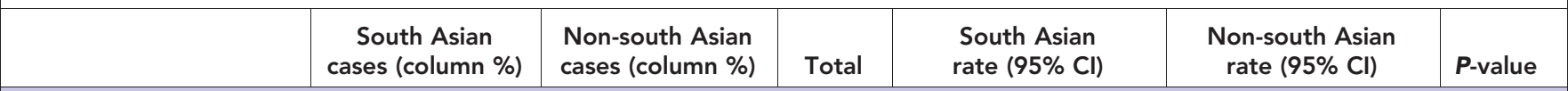

Diagnostic group

\begin{tabular}{|l|c|}
\hline Leukaemia & $66(24)$ \\
\hline Lymphoma & $71(26)$ \\
\hline CNS tumours & $37(13)$ \\
\hline Other solid tumours & $101(37)$
\end{tabular}

Age at diagnosis, years (\%)

\begin{tabular}{|l|c|c|c|c|c|c|}
\hline $0-4$ & $66(24)$ & $650(20)$ & 716 & $194.7(147.7-241.7)$ & $192.6(177.8-207.4)$ & 0.918 \\
\hline $5-9$ & $40(15)$ & $364(11)$ & 404 & $125.9(86.8-165.0)$ & $102.6(92.0-113.1)$ & 0.225 \\
\hline $10-14$ & $37(13)$ & $375(12)$ & 412 & $125.8(85.2-166.3)$ & $105.0(94.3-115.6)$ & 0.280 \\
\hline $15-19$ & $42(15)$ & $463(14)$ & 505 & $135.5(94.5-176.6)$ & $131.0(119.0-143.0)$ & 0.723 \\
\hline $20-24$ & $41(15)$ & $597(18)$ & 638 & $125.2(86.6-163.7)$ & $161.0(148.1-174.0)$ & 0.181 \\
\hline $25-29$ & $49(18)$ & $810(25)$ & 859 & $169.9(122.0-217.8)$ & $217.1(202.1-232.1)$ & 0.146 \\
\hline Gender & \multicolumn{7}{|l|}{} \\
\hline Male & $159(58)$ & $1995(61)$ & 2154 & $170.5(143.8-197.3)$ & $183.2(175.1-191.2)$ & 0.555 \\
\hline Female & $116(42)$ & $1264(39)$ & 1380 & $119.9(97.9-141.8)$ & $119.3(112.7-125.9)$ & 0.744 \\
\hline Total & 275 & 3259 & 3534 & $145.2(127.9-162.5)$ & $151.4(146.2-156.6)$ & 0.801 \\
\hline
\end{tabular}



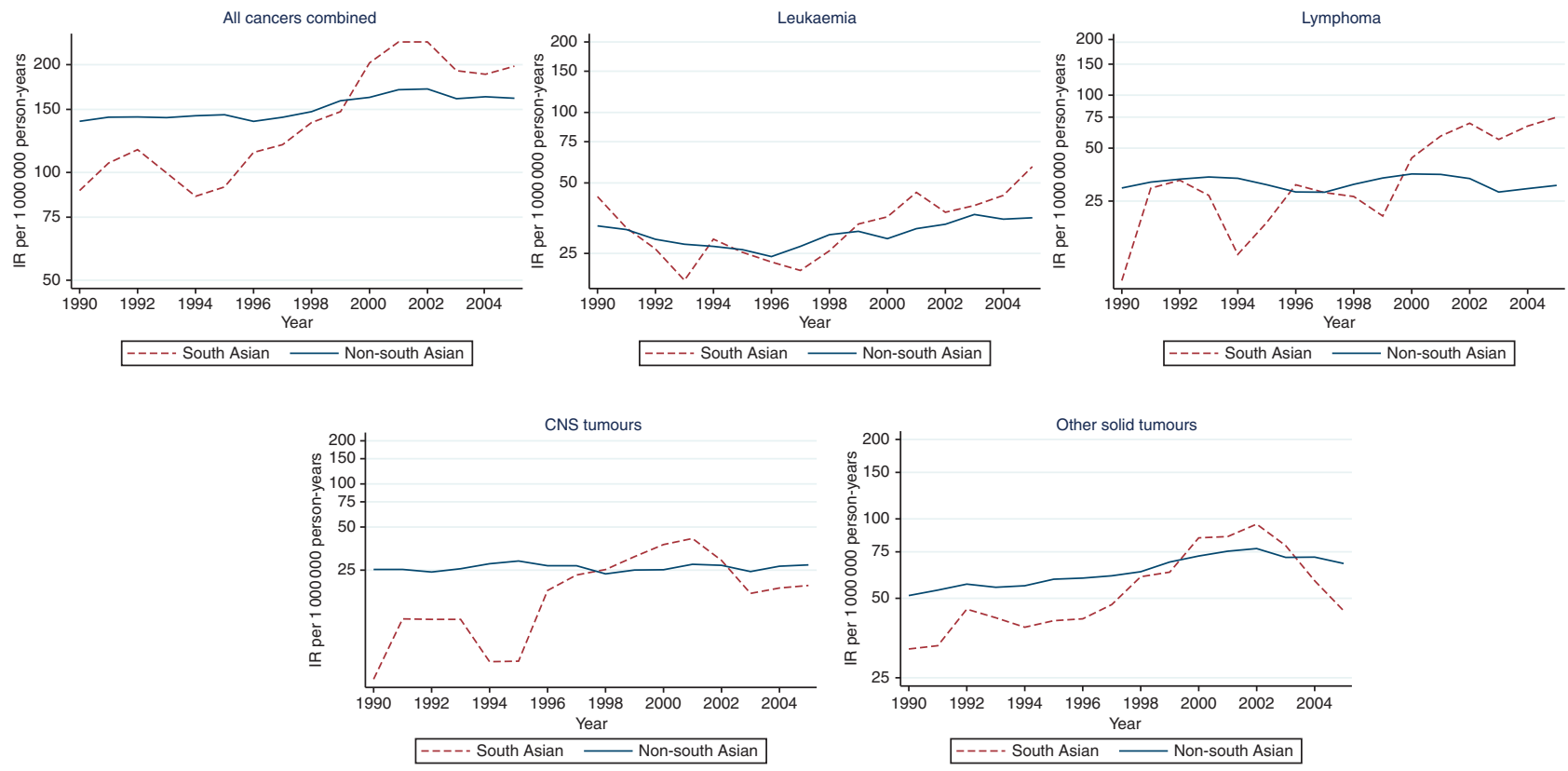

Figure 1. Smoothed (3-year moving average) age- and sex-standardised incidence rates (IR) by ethnic group in Yorkshire, UK (1990-2005).

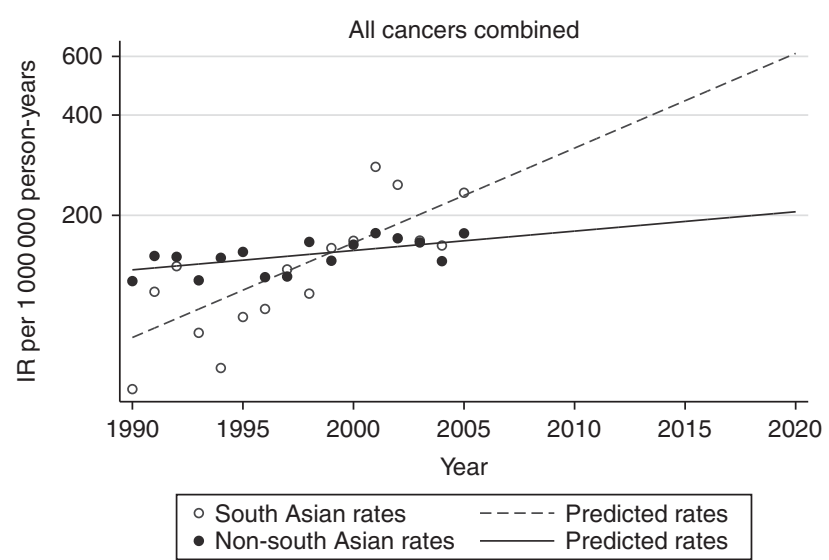

Figure 2. Age- and sex-standardised incidence rates (IR) and predicted rates by ethnic group for all cancers combined.
The incidence rate among south Asians and non-south Asians is 145.2 (95\% CI 127.9-162.5) and 151.4 (95\% CI 146.2-156.6), respectively. Table 1 shows the number of cases and incidence rates for south Asians and non-south Asians by diagnostic group, age group and gender.

\section{Temporal trends}

Figure 1 displays incidence trends between 1990 and 2005 for all cancers combined and each diagnostic group using smoothed 3-year moving averages (MA).

Figure 2 shows the age- and sex-standardised incidence rates for south and non-south Asians for all cancers combined alongside predicted incidence rates from 2005 to 2020 . If the AAPC continues to increase at a constant rate, the incidence of cancer among south Asians is set to increase almost threefold from 2005 onwards to be approximately 600 per million compared with 200 per million for non-south Asians by 2020. 